\title{
Infrared Emission from AGN
}

\author{
D. B. Sanders \\ Institute for Astronomy, University of Hawaii, 2680 Woodlawn Drive, \\ Honolulu, HI 96822
}

\begin{abstract}
Infrared observations of complete samples of active galactic nuclei (AGN) have shown that a substantial fraction of their bolometric luminosity is emitted at wavelengths $\sim 8-1000 \mu \mathrm{m}$. In radio-loud and Blazar-like objects much of this emission appears to be direct nonthermal synchrotron radiation. However, in the much larger numbers of radio-quiet AGN it is now clear that thermal dust emission is responsible for the bulk of radiation from the near-infrared through submillimeter wavelengths. Luminous infrared-selected AGN are often surrounded by powerful nuclear starbursts, both of which appear to be fueled by enormous supplies of molecular gas and dust funneled into the nuclear region during the strong interaction/merger of gas rich disks. All-sky surveys in the infrared show that luminous infrared AGN are at least as numerous as optically-selected AGN of comparable bolometric luminosity, suggesting that AGN may spend a substantial fraction of their lifetime in a dust-enshrouded phase. The space density of luminous infrared AGN at high redshift may be sufficient to account for much of the X-Ray background, and for a substantial fraction of the far-infrared background as well. These objects plausibly represent a major epoch in the formation of spheroids and massive black holes (MBH).
\end{abstract}

\section{Introduction}

Infrared observations ${ }^{1}$ of AGN historically have lagged behind observations at shorter and longer wavelengths, therefore it is not surprising that much of the literature is still biased toward studies of radio and optical/X-Ray selected AGN. While radio-loud objects and the highly variable optical/X-Ray sources (e.g. Blazars, OVVs) provide an opportunity for studying the physics of AGN, they draw attention away from the much larger fraction of radio-quiet and dustenshrouded sources. These dusty AGN appear to hold important clues for understanding the origin and evolution of all AGN, and the relation of AGN to other classes of extragalactic objects.

\footnotetext{
1 "Infrared" is used here to include rest-frame emission over the broad wavelength range 8$1000 \mu \mathrm{m}$ (i.e. the mid-infrared, far-infrared and submillimeter, but not the near-infrared). Definitions for observed quantities such as $L_{\text {ir }}, L_{\mathrm{B}}$, etc. are taken from Table 1 of the review by Sanders \& Mirabel (1996). $H_{\mathrm{o}}=75$ and $q_{\mathrm{o}}=0$ is assumed throughout this article.
} 
This review focuses on the relatively large body of infrared continuum data ${ }^{2}$ that is now available for complete samples of nearby AGN, and stresses new results that have been published since the previous IAU Symposium on AGN in Geneva, 1993 ("Multiwavelength Continuum Observations of AGN", S159). A major highlight of the most recent work is the clear identification of a large population of dust-enshrouded AGN that may be more numerous than optically selected AGN in the Universe.

In reviewing the infrared continuum properties of AGN, it is instructive to trace the highlights of infrared studies from the first mid- and far-infrared measurements of selected nearby targets in the late 60 's and 70 's, through to the latest spacecraft results that bear directly on the AGN population in the more distant Universe. Infrared observations took a great step forward following the all-sky surveys carried out by the Infrared Astronomical Satellite (IRAS), and it seems natural to divide our initial discussion accordingly, and then to show how infrared studies of AGN in the local Universe can help in understanding the AGN population at high redshift.

\section{Pre-IRAS: A Brief Historical Review, 1968-83}

The pioneering infrared observations of Low \& Kleinmann (1968), and Kleinmann \& Low $(1970 \mathrm{a}, \mathrm{b})$, followed by more accurate photometry by Rieke \& Low (1972), made it clear that strong infrared emission could dominate the spectral energy distributions (SEDs) of Seyfert galaxies, and even singled out a class of objects with "ultra-high" infrared luminosities that rivaled the bolometric luminosity of QSOs. The first clear evidence that the mid-infrared emission from most Seyferts might not be direct synchrotron radiation was provided by new $10 \mu \mathrm{m}$ data for NGC1068 which showed lack of variability (Stein et al. 1974), plus an extended source (Becklin et al. 1973). The infrared spectrum appeared to be better explained by models of thermal reradiation from dust (e.g. Rees et al. 1969; Burbidge \& Stein 1970).

Mid-infrared ground-based photometry of large samples of Markarian Seyferts and starbursts (Rieke \& Low 1975; Neugebauer et al. 1976), Seyfert galaxies (Rieke 1978), plus mid- and far-infrared observations of a few nearby Seyferts with the Kuiper Airborne Observatory (Harper \& Low 1973; Telesco \& Harper 1980 ) proved that "infrared-excess" was a common property of Seyferts as well as starburst galaxies, and with the possible exception of radio-loud objects and QSOs, that this emission could indeed be understood in terms of thermal emission from dust. Rieke (1978) perhaps summarized it best (from a study of 50+ Markarian Seyferts) by stating that "strong infrared excess is a virtually universal characteristic of these sources. ...the infrared continuum of a number of type $1 \mathrm{~s}$ and most type $2 \mathrm{~s}$ is dominated by thermal reradiation by dust", while also pointing out that the strength of the infrared excess was correlated with the

\footnotetext{
${ }^{2}$ Mid- and far-infrared spectroscopy of AGN is not covered in this review. Until very recently, such data were available for only a small number of optically selected targets. However, data for a larger number of nearby AGN are now available from the Infrared Space Observatory (ISO), and the reader is referred to articles by $\mathrm{O}$. Laurent and J. Clavel at this conference, and to the excellent paper by Genzel et al. (1998).
} 
strength of the reddening (as measured by $\mathrm{H} \alpha / \mathrm{H} \beta$ ), and that the proportionality between the $10 \mu \mathrm{m}$ and $21 \mathrm{~cm}$ fluxes noted earlier (e.g. Rieke \& Low 1972) was confirmed.

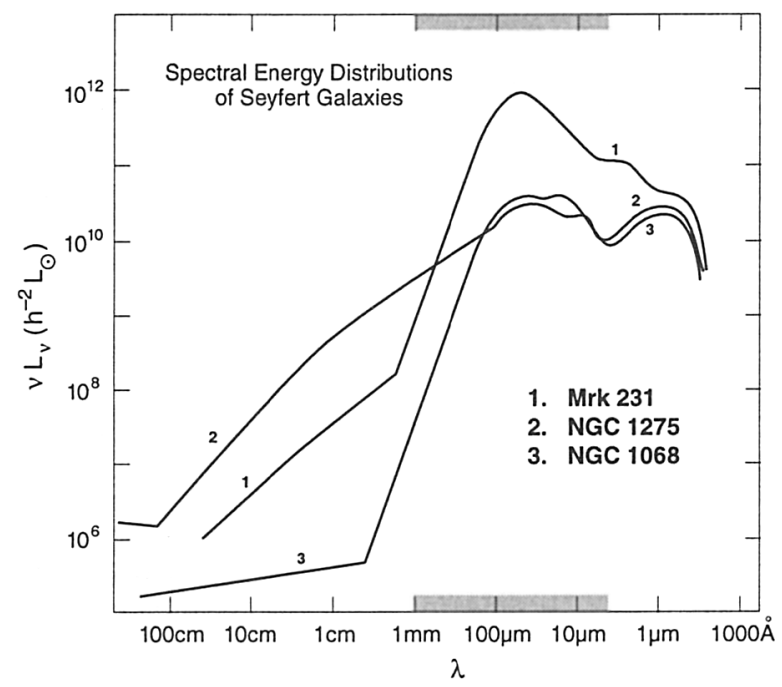

Figure 1. Spectral energy distributions from UV to radio wavelengths for three well-known AGN: the Sy1 galaxy Mrk231, the Sy2 galaxy NGC1068, and the Sy2 powerful radio galaxy NGC1275 (Perseus A).

Figure 1 shows SEDs using the most recent radio-to-UV continuum data for three "classic" Seyferts, which were among the first AGN observed in the mid- and far-infrared. The steep submillimeter spectral index, $\alpha>2.5$ (where $\left.f_{\nu} \propto \nu^{\alpha}\right)$, for radio-quiet objects confirms earlier suggestions that the "infrared bump" at wavelengths $\sim 5-500 \mu \mathrm{m}$ is due to thermal emission from dust. Only in the radio-loud sources such as NGC1275 (Perseus A) is there any reason to believe that a substantial portion of the infrared emission is simply the short wavelength extension of the non-thermil emission seen at radio-to-millimeter wavelengths (e.g. Edelson \& Malkan 1986).

\section{Post-IRAS: Infrared Properties of Optical Samples of AGN}

IRAS was the first telescope with sufficient sensitivity to detect large numbers of extragalactic sources at mid-and far-infrared wavelengths (Neugebauer et al. 1984). IRAS surveys of optically selected Seyfert galaxies (Miley et al. 1985) and QSOs (Neugebauer et al. 1985, 1986) confirmed that active galaxies could be strong infrared emitters; most optically selected AGNs had ratios $L_{\mathrm{ir}} / L_{\mathrm{B}}$ in the range 0.2 to 1.0 with higher values in only a small number of objects. 


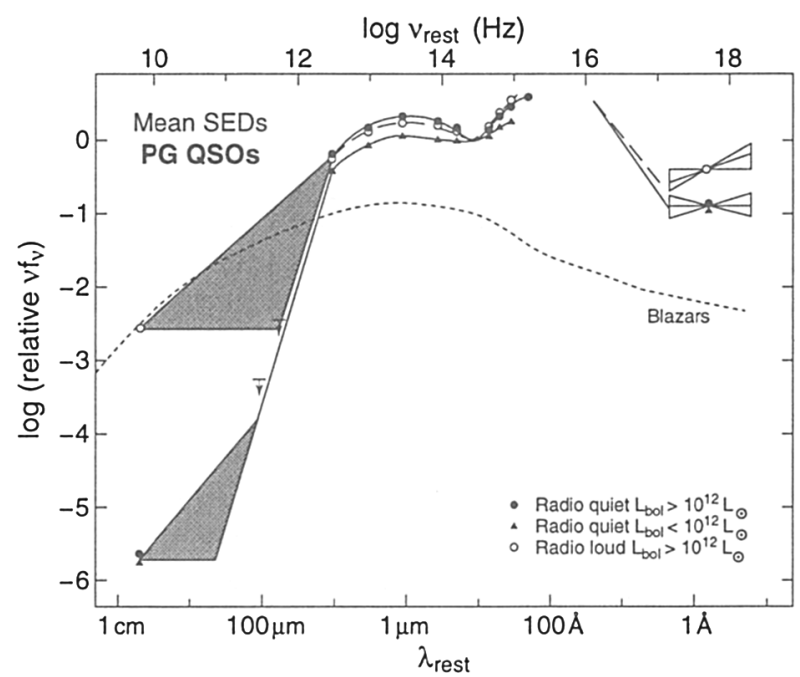

Figure 2. Mean spectral energy distributions from X-ray to radio wavelengths for optically selected radio-loud and radio-quiet QSOs (Sanders et al. 1989) from the Palomar-Green Bright QSO Survey (Schmidt \& Green 1983), and for Blazars (Impey \& Neugebauer 1988).

\subsection{QSOs}

A more complete accounting of the infrared properties of optically selected Syls and QSOs is given by the data in Figure 2. The interpretation by Sanders et al. (1989) was that the gross shape of the SEDs between $3000 \AA$ and $300 \mu \mathrm{m}$ is remarkably similar for all QSOs (except the flat-spectrum radio-loud quasars like $3 \mathrm{C} 273$ ) and that this can broadly be interpreted by two broad components of thermal emission; the "big blue bump" representing $10^{5}-10^{6} \mathrm{~K}$ thermal emission from an accretion disk, and an "infrared bump" made up of reradiation from dust in a distorted disk extending from $\sim 0.1 \mathrm{pc}$ to more than $1 \mathrm{kpc}$.

Only for flat-spectrum radio-loud QSOs is there good evidence that much of the infrared emission is probably direct non-thermal emission from the central AGN. These objects also tend to exhibit variability on relatively short time-scales (hours to weeks). In the highly variable Blazars there is strong evidence that infrared emission is truly just part of a single non-thermal spectral component from millimeter to optical wavelengths (see Figure 2). The range of variability in both the spectral index and flux density for Blazars increases with decreasing wavelength, with the variability in the far-infrared being less than half that observed in the optical. However, a substantial fraction of Blazars, when in their minimum variable state, also show evidence for underlying emission lines and thermal infrared components, suggesting that these objects may still contain substantial amounts of dust. 


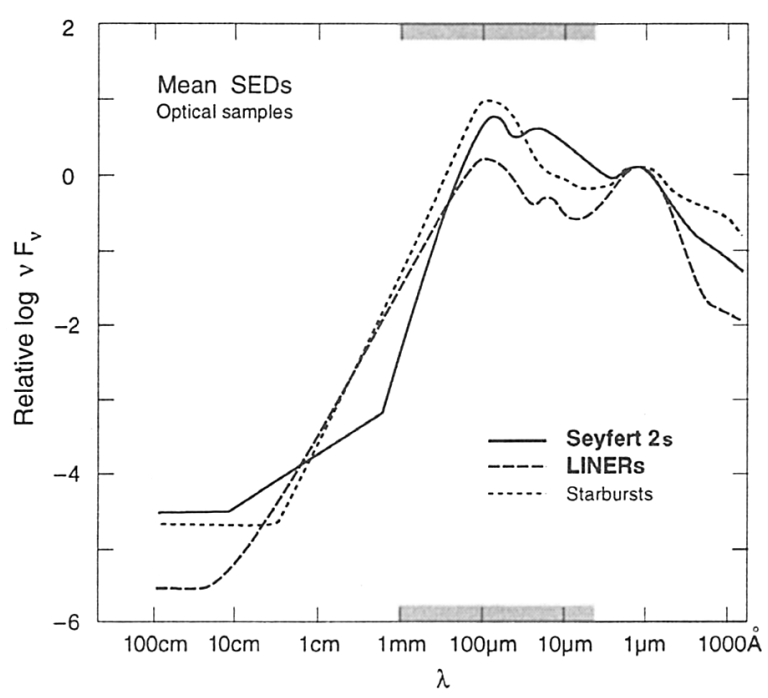

Figure 3. Mean spectral energy distributions from X-ray to radio wavelengths (normalized to $\log \nu F_{\nu}(7700 \AA) \equiv 0$ ) for optically selected NLAGN (Sy2s and LINERs) compared with luminous starbursts (adapted from Schmitt et al. 1997).

\subsection{Narrow-line AGN}

A more complete accounting of the infrared properties of optically selected narrow-line AGN (NLAGN) is given by the data in Figure 3, which suggest a larger mean infrared excess $\left(\equiv L_{\text {ir }} / L_{\mathrm{B}}\right)$ than previously assumed; the mean value is $\sim 1$ for LINERs and $\sim 5$ for Sy2s. Both Seyferts and LINERs have a farinfrared peak at $\sim 60-200 \mu \mathrm{m}$ characteristic of relatively "cool" dust $\left(T_{\text {dust }} \sim 25-\right.$ $50 \mathrm{~K}$ ), with the strength of the infrared emission in Sy2s actually being somewhat larger than that observed in starbursts (!), primarily due to added emission from a second "warm" ( $\left.T_{\text {dust }} \sim 100-500 \mathrm{~K}\right)$ dust peak in Sy2s at wavelengths $\sim 5-50 \mu \mathrm{m}$.

\section{Infrared Selected AGN}

The true extent of infrared excess exhibited by all types of extragalactic objects was only begun to be realized following extensive ground-based follow-up studies of complete infrared selected samples. deGrijp et al. (1985) found that searches based on "warm" infrared colors $\left(f_{25} / f_{60} \gtrsim 0.3\right)$ could be useful for discovering new infrared-luminous AGN, a technique that appeared to be motivated by the shape of the infrared spectrum of the Sy2 galaxy NGC1068 (Telesco \& Harper 1980), and the discovery of a similar "warm" component in the broadline, infrared-luminous radio galaxy 3C290.3 (Miley et al. 1984). These infraredselected AGN had a mean ratio of $L_{\text {ir }} / L_{\mathrm{B}} \sim 10$ and included a few objects with ratios as large as $\sim 30-50$. deGrijp et al. (1985) suggested that the true space density of AGNs could be a factor of two larger than previously assumed (with 


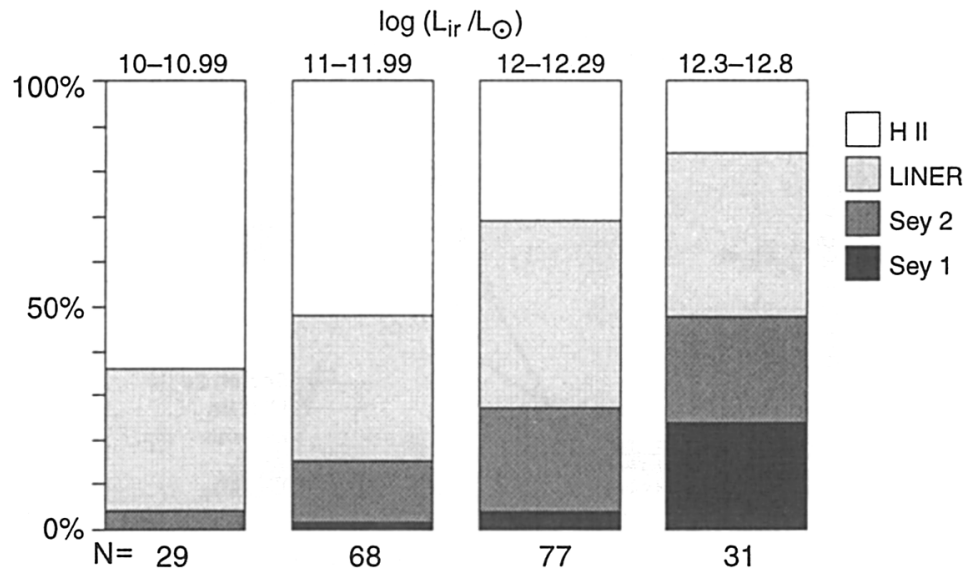

Figure 4. The optical spectral classification of flux-limited $(60 \mu \mathrm{m})$ samples of infrared selected galaxies versus infrared luminosity (Veilleux et al. 1999).

the majority of the new infrared-selected objects being a mixture of Sy2s and LINERs).

The first infrared-selected "bonifide" QSOs ${ }^{3}$ were also found during colorselected searches of the IRAS database at the faintest flux levels (Beichman et al. 1986; Vader \& Simon 1987; Low et al. 1988; Sanders et al. 1988b). Additionally, the most luminous infrared sources (with $L_{\mathrm{ir}} \sim 10^{13} L_{\odot}$ ), all of which were found to be dusty Sy2s in direct emission (e.g. Kleinmann \& Keel 1987; Hill et al. 1987; Frogel et al. 1989; Cutri et al. 1994) were subsequently shown to be obscured infrared QSOs (i.e. Sy1s) in polarized light (e.g. Hines et al. 1995). Whether all infrared selected Sy2s harbor obscured Sy1s is not yet clear. However, it seems plausible that "unified models" invoked to understand the polarization properties of optically selected AGN (e.g. Antonucci \& Miller 1985), which suggest that the observed spectral type (Sy1 vs. Sy2) depends largely on the orientation of a circumnuclear dust torus to the line of sight (see Antonucci 1993 for a more complete review) could play a major role in infrared-selected AGN. Models with even larger dust shrouds (>100 pc) where the obscuring material covers most of the sky as seen from the central source (e.g. Fabian et al. 1998) may also need to be invoked to account for the objects with the largest infrared excess. 


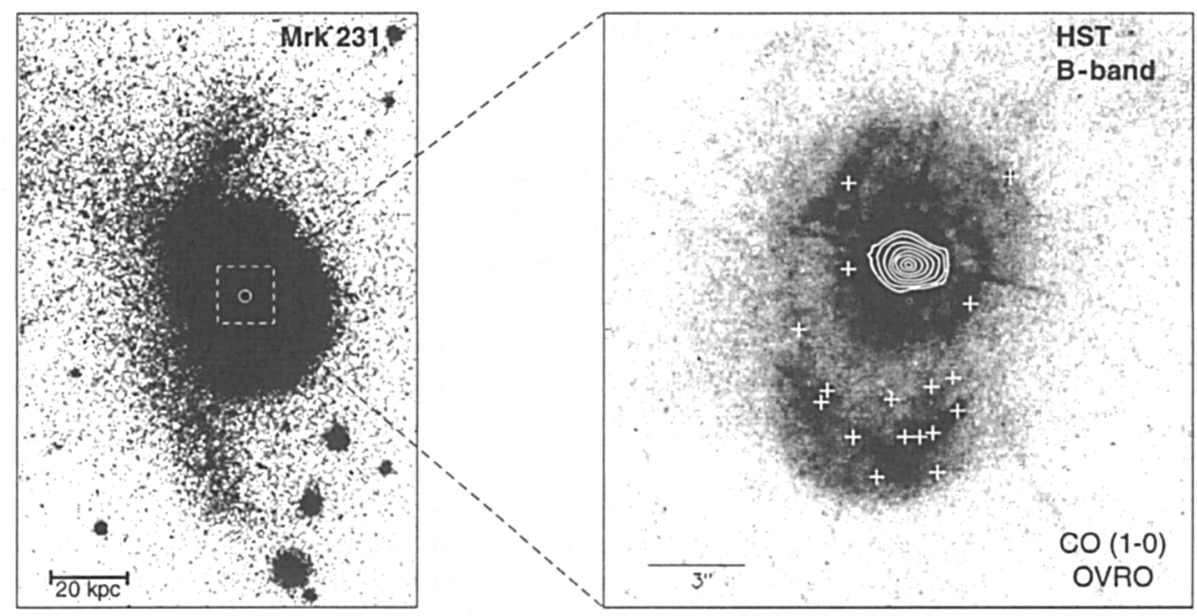

Figure 5. The advanced merger/ULIG/QSO Mrk 231 - Left panel: optical image (Sanders et al. 1987) and CO contour (Scoville et al. 1989). Right panel: HST B-band image and identified stellar clusters ('t') from Surace et al. (1998). The high resolution CO contours are from Bryant \& Scoville (1997).

\subsection{AGN versus Infrared Luminosity}

Perhaps the most important spectroscopic result from studies of flux-limited samples of extragalactic infrared objects is the increasing fraction of AGN among the most luminous sources. It is clear from Figure 4 that the fraction of Seyferts increases systematically with increasing $L_{\mathrm{ir}}$, to where Seyferts account for nearly half of all objects at the highest infrared luminosities. The ratio of Sy1s to Sy2s also increases to the point where both are $\sim 25 \%$ of the total number of objects with $L_{\text {ir }}>10^{12.3} L_{\odot}$. Whereas from optical surveys alone it had been thought that Sy2s were very rare at high bolometric luminosities, it now seems clear that high luminosity Sy2s were simply hiding as a subset of the most luminous infrared selected galaxies. Likewise for luminous H II galaxies, although the fraction of H II galaxies diminishes to $\$ 25 \%$ for ultraluminous infrared galaxies (ULIGs) at $L_{\text {ir }}>10^{12} L_{\odot}$. LINERs appear to remain constant at $\sim 1 / 3$ of the sample at all $L_{\mathrm{ir}}$, and although LINERs have sometimes been lumped together with NLAGN, recent evidence suggests that the emission lines in many of these infrared-selected LINERs may be powered primarily by shocks and superwinds from massive stars (e.g. Veilleux et al. 1999). 


\section{The Starburst-AGN Connection}

There is increasing direct evidence that powerful circumnuclear starbursts and AGN may be intimately related. An excellent review of this subject can be found in the Taipei Workshop - Relationships between AGN and Starburst Galaxies (1992). For the current discussion, the case of Mrk231 (Figure 5) is instructive. Mrk231, like other ULIGs, contains a large population of relatively unobscured luminous star clusters at galactocentric radii $\sim 0.5-3 \mathrm{kpc}$ (Surace et al. 1998). The inner $1 \mathrm{kpc}$ region still contains an enormous supply of gas and dust, much of which may be arranged in a subkiloparsec disk (Carilli et al. 1998). A partial face-on orientation for this disk may be the explanation for why we can see the redenned Sy1 nucleus.

It has been suggested that both the intense circumnuclear starburst and the AGN currently contribute approximately equally to the bolometric infrared luminosity in Mrk231 (D. Weedman and H.E. Smith, this conference). However, the nature of the dominant power source for ULIGs continues to be the subject of great debate (e.g. Ultraluminous Galaxies: Monsters or Babies, Ringberg Workshop, 1998) with arguments favoring both circumnuclear starbursts and AGN as well as nearly equal mixtures of the two phenomena. Extensive multiwavelength spectroscopic studies are currently underway in an attempt to resolve the issue (e.g. Genzel et al. 1998), but due to heavy dust obscuration it is not yet clear which process dominates in all objects.

\subsection{The Origin and Evolution of Infrared-luminous AGN}

Extensive ground-based observations of complete samples of infrared-selected galaxies now clearly show that strong interactions/mergers of gas-rich spirals play a dominant role in triggering the most luminous infrared systems (see the review by Sanders \& Mirabel 1996). The fraction increases from $\sim 30 \%$ at $L_{\text {ir }}=$ $10^{11} L_{\odot}$ to $\gtrsim 95 \%$ for ULIGs at $L_{\text {ir }}>10^{12} L_{\odot}$ (e.g. Sanders et al. 1988a; Mirabel et al. 1990; Kim 1995; Murphy et al. 1996; Clements et al. 1996). Prominent tidal tails, similar to what is seen for Mrk231 (Figure 5), are indeed found in all nearby ULIGs, suggesting that the mergers involve two relatively large gas-rich spirals. Figure 6 illustrates the ubiquitous tidal tails, and in some cases double nuclei, that can still be detected in deep images of even more distant luminous infrared objects, in this case three optically selected AGN.

Strong interactions/mergers of gas-rich spirals appear to be extremely efficient at funneling large amounts of gas into the merger nuclei (e.g. Barnes \& Hernquist 1992; Mihos \& Hernquist 1994). Kormendy \& Sanders (1992) have summarized the evidence that these objects are elliptical galaxies in formation. Intense starbursts are clearly involved in producing the bulk of the infrared luminosity throughout much of the initial stages of this process, and may continue to do so through the most intense infrared phase, although it seems reasonable to assume that during the peak infrared phase (which is close in time to when the two nuclei merge and also corresponds to the most compact concentration of gas in the nuclear region), conditions would be most optimum for building and

${ }^{3}$ (i.e. Syls with $L_{\mathrm{ir}}>10^{12} L_{\odot}$; equivalent to the bolometric luminosity of optically selected QSOs with $\left.M_{\mathrm{B}}<-23\right)$ 


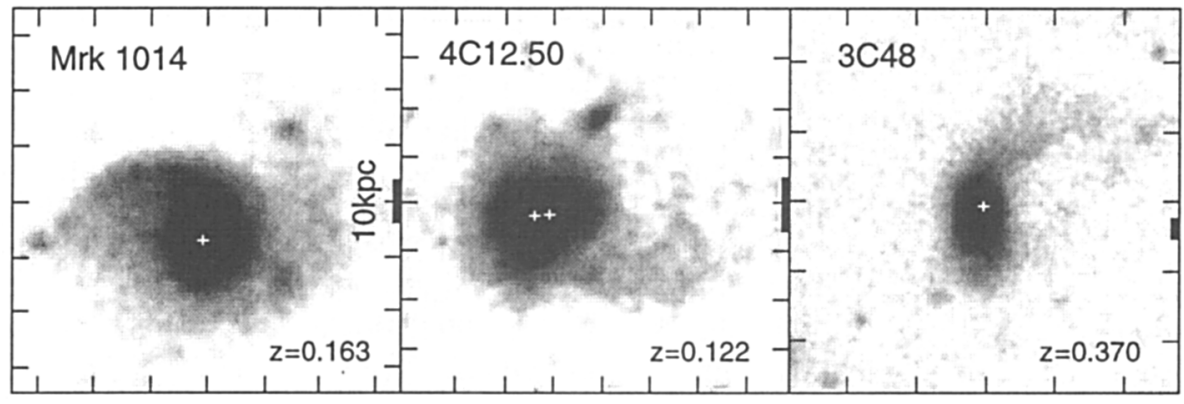

Figure 6. Optical images of infrared-excess, optically selected QSOs, powerful radio galaxies, and infrared selected QSOs (MacKenty \& Stockton 1984; Kim 1995; Stockton \& Ridgway 1991). The 't' sign indicates the position of putative optical nuclei. Tick marks are at $5^{\prime \prime}$ intervals and the scale bar represents $10 \mathrm{kpc}$. All three objects exhibit strong nuclear concentrations of molecular gas, with typically $\sim 10^{10} M_{\odot}$ concentrated at galactocentric radii $\leqslant 1 \mathrm{kpc}$ (Sanders et al. 1988c; Mirabel et al. 1989; Scoville et al. 1989).

fueling an AGN, and that the AGN may rival if not dominate the luminosity output of the system. Mrk231 and most other ULIGs would seem to be at this stage.

Eventually, powerful superwinds (e.g. Armus et al. 1989), that are indeed observed in ULIGs, may clear away much of the surrounding nuclear dust shroud. It seems reasonable that this housecleaning process could terminate much of the circumnuclear starburst (as it already may have in the 1-3 kpc annulus of Mrk231), but that the fueling of the AGN may continue due to the strong selfgravitation of the inner accretion disk surrounding the AGN. This later period may still be marked by infrared excess determined by conditions on much smaller scales such as the thickness, orientation, and opening angle of the dust and gas torus thought to surround the AGN. Eventually the "big blue bump" normally associated with optically selected QSOs should emerge, as has happened already in the case of Mrk1014 and 3C48 (Figure 6). This scenario would seem to provide a plausible explanation for a correlation between black hole mass and bulge mass as recently found in nearby ellipticals (Kormendy \& Richstone 1995) since the mass of both the black hole and spheroid may show similar dependence on the total mass of gas funneled into the merger nucleus.

\section{Deep Infrared and X-Ray Surveys: AGN and the Distant Uni- verse}

There is now good evidence that both ULIGs and NLAGN may be sufficiently numerous at high redshift $(z>1)$ to account for the infrared background and the $\mathrm{X}$-Ray background respectively. There is also increasing circumstantial evidence that suggests NLAGN may be a substantial subset of these high-redshift ULIGs, 
which if true, would suggest that a major epoch in the formation of spheroids and $\mathrm{MBH}$ may have finally been discovered.

The evidence from the X-Ray side is as follows. Deep X-Ray surveys with ROSAT are consistent with a space density that evolves as steeply as $(1+$ $z)^{5}$ for high-luminosity AGN out to at least $z \sim 2$ with a constant value at higher redshift (e.g. Hassinger et al. 1998). These sources are sufficiently numerous to account for nearly all of the observed X-Ray background; however, the shape of the X-Ray background spectrum implies that most of these AGN are heavily absorbed (e.g. Fabian \& Barcons 1992; Boyle et al. 1995; Almaini et al. 1998). These "narrow-line X-Ray galaxies" (NLXGs: Hassinger 1996) have recently been characterized by Maiolino et al. (1998) as having "extremely heavy obscuration along the line of sight, $\left(N_{\mathrm{H}}>10^{25} \mathrm{~cm}^{-2}\right)$ in most cases".

The evidence from the infrared side is as follows. Evidence for space density evolution as steep as $(1+z)^{5}$ is consistent with a series of studies at varying far-infrared flux levels and wavelength bands. In the mid- and far-infrared, the deepest surveys carried out by IRAS (e.g. Hacking \& Houck 1987; Lonsdale \& Hacking 1989; Gregorich et al. 1995; Kim \& Sanders 1998), and more recently the surveys with ISO (e.g. Taniguchi et al. 1997; Kawara et al. 1998; Aussel et al. 1998; Puget et al. 1998) are consistent with evolution at least as steep as $(1+z)^{5}$ out to $z \sim 1$. Within the past year, submillimeter surveys with the Submillimeter Common User Bolometer (SCUBA) on the James Clerk Maxwell Telescope (Smail et al. 1997; Hughes et al. 1998; Barger et al. 1998; Eales et al. 1998) have revealed a substantial population of ULIGs, consistent with steep evolution out to at least $z \sim 2-3$, and with constant space density at higher redshift. These high redshift ULIGs, which are almost certainly the high- $z$ extension of the sources detected by $I R A S$ and $I S O$, are sufficiently numerous to account for all of the far-infrared/submillimeter background (e.g. Barger et al. 1999).

To test the relationship between ULIGs and NLXGs at high redshift requires sufficiently sensitive X-Ray and far-infrared/submillimeter surveys in overlapping regions of the sky. These data should be available within the next few years from new X-Ray satellites and submillimeter interferometers. For now we can only note the interesting result that all of the ULIGs uncovered by IRAS at $z>0.4$ (all of which have $L_{\mathrm{ir}} \sim 10^{13} L_{\odot}$ ) show direct evidence for powerful AGN (e.g. Kleinmann \& Keel 1987; Rowan-Robinson et al. 1991; Cutri et al. 1994), as does the first identified SCUBA source (Ivison et al. 1998). It would thus appear that the relationship shown in Figure 4 indeed continues to higher luminosities and to higher redshift.

\section{Conclusions}

Infrared observations have shown clearly that thermal emission plays an important, and often dominant, role in the total luminosity output of most AGN. Although in some objects - most notably the flat-spectrum, radio-loud AGN and the highly variable Blazars - a substantial fraction of the observed infrared emission appears to be direct non-thermal synchrotron radiation, the much larger number of radio-quiet Sy1s and NLAGN appear to have infrared SEDs dominated by thermal emission from dust. 
The discovery by IRAS of a substantial population of infrared-selected AGN suggests that a substantial fraction of the energy produced by accretion may be absorbed by dust. Ground-based observations of these dusty objects show that powerful circumnuclear starbursts $(r \lesssim 1 \mathrm{kpc})$ are often closely linked with the building and fueling of AGN, and that the most luminous sources (ULIGs) are often associated with strongly interacting/merger galaxies.

There is now strong evidence that the space density of ULIGs was much larger in the past $(z>1)$, and that they may account for a large fraction, if not all, of the far-infrared/submillimeter background. If the trend of increasing AGN fraction versus increasing infrared luminosity observed for local ULIGs continues to high redshift, then dust-enshrouded AGN may account for much of the X-Ray background as well. This large population of high-redshift ULIGs may represent an important stage in the formation of both spheroids and massive black holes.

Acknowledgments. I am grateful to Karen Teramura for assistance in preparing the figures, and to JPL contract no. 961566 for partial financial support.

\section{References}

Almani, O., et al. 1998, Astr.Nachr., 319, 55

Antonucci, R. 1993, ARA\&A, 31, 473

Antonucci, R. \& Miller, J.S. 1985, ApJ, 297, 621

Armus, L., Heckman, T.M., \& Miley, G.K. 1989, ApJ, 347, 727

Aussel, H., Cesarsky, C.J., Elbaz, D., \& Starck, J.L. 1998, A\&A, in press

Barger, A.J., Cowie, L.L., \& Sanders, D.B. 1999, ApJ, submitted

Barger, A.J., et al. 1998, Nature, 394, 248

Barnes, J.E., \& Hernquist, L. 1992, ARA\&A, 30, 705

Becklin, E.E., Matthews, K., Neugebauer, G., \& Wynn-Williams, G.C. 1973, ApJ, 186, L69

Beichman, C.A., et. al. 1986, ApJ, 308, L1

Bryant, P.M., \& Scoville, N.Z. 1996, ApJ, 457, 678

Burbidge, G.R., \& Stein, W.A. 1970, ApJ, 160, 573

Carilli, C.L., Wrobel, J.M., \& Ulvestad, J.S. 1998, A\&A, 115, 928

Clements, D.L., Sutherland, W.J., McMahon, R.G., \& Saunders, W. 1996, MNRAS, 279,477

Cutri, R.M., et al. 1994, ApJ, 424, L65

de Grijp, Miley, G.K., Lub, J., \& de Jong, T. 1985, Nature, 314, 240

Eales, S., et al. 1998, ApJ, in press

Edelson, R.A., \& Malkan, M.A. 1986, ApJ, 308, 59

Fabian, A.C., \& Barcons, X. 1992, ARA\&A, 30, 429

Fabian, A.C., \& Barcons, X., Almaini, O., \& Iwasawa, I. 1998, MNRAS, 297, L11

Frogel, J.A., Gillett, F.C., Tendrup, D.M., \& Vader, J.P. 1989, ApJ, 343, 672

Genzel, R., et al. 1998, ApJ, 498, 579 
Gregorich, D.T., Neugebauer, G., Soifer, B.T., Gunn, J.E., \& Herter, T.L. 1995, AJ, 110, 259

Hacking, P.B., \& Houck, J.R. 1987, ApJS, 63, 311

Harper, D.A., \& Low, F.J. 1973, ApJ, 182, L89

Hassinger, G. 1996, A\&AS, 120, 607

Hassinger, G., et al. 1998, A\&A, 329, 482

Heckman, T.M. 1980, A\&A, 87, 152

Hill, G.J., Wynn-Williams, C.G., \& Becklin, E.E. 1987, ApJ, 316, L11

Hines, D.C., et al. 1995, ApJ, 450, L1

Hughes, D.H., et al. 1998, Nature, 394, 241

Impey, C., \& Neugebauer, G. 1988, AJ, 95, 307

Ivison, R., et al. 1998, MNRAS, 298, 583

Kawara, K., et al. 1998, A\&A, 336, L9

Kim, D.-C., et al. 1995, PhD Thesis, University of Hawaii

Kim, D.-C., \& Sanders, D.B. 1998, ApJS, 119, 41

Kleinmann, D.E., \& Low, F.J. 1970a, ApJ, 159, L165

Kleinmann, D.E., \& Low, F.J. 1970b, ApJ, 161, L203

Kleinmann, S.G., \& Keel, W.C. 1987, in Star Formation in Galaxies, ed. C.J. Lonsdale-Persson, Wash. DC: US GPO, 559

Kormendy, J., \& Richstone, D. 1995, ARA\&A, 33, 581

Kormendy, J., \& Sanders, D.B. 1992, ApJ, 390, L53

Lonsdale, C.J., \& Hacking, P. 1989, ApJ, 339, 712

Low, F.J., Huchra, J.P., Kleinmann, S.G., Cutri, R.M. 1988, ApJ, 327, L41

Low, F.J., \& Kleinmann, D.E. 1968, AJ, 73, 868

MacKenty, J.W., \& Stockton, A. 1984, ApJ, 283, 64

Maiolino, R., et al. 1998, A\&A, 338, 781

Mihos, J.C., \& Hernquist, L. 1994, ApJ, 431, L9

Miley, G.K., Neugebauer, G., \& Soifer, B.T. 1985, ApJ, 293, L11

Miley, G.K., et al. 1984, ApJ, 278, L79

Mirabel, I.F., Booth, R.S., Garay, G., , L.E.B., \& Sanders, D.B. 1990, A\&A, 236,327

Mirabel, I.F., Sanders, D.B., \& Kazès, I. 1989, ApJ, 340, L9

Multiwavelength Continuum Emission of AGN 1994, eds. T.J.-L. Courvoisier, A. Blecha (Dordrecht: Kluwer)

Murphy, T.W., et al. 1996, AJ, 111, 1025

Neugebauer, G., Becklin, E.E., Oke, J.B., \& Searle, L. 1976, ApJ, 205, 29

Neugebauer, G., Soifer, B.T., \& Miley, G.K. 1985, ApJ, 295, L27

Neugebauer, G., Soifer, B.T., \& Miley, G.K., \& Clegg, P.E. 1986, ApJ, 308, 815

Neugebauer, G., et al. 1984, ApJ, 278, L1

Puget, J.-L., et al. 1998, A\&A, in press

Rees, M.J., Silk, J.I., Werner, M.W., \& Wickramasinghe, N.C. 1969, Nature, 223, 37 
Relationships Between AGN and Starburst Galaxies 1992, ed. A.V. Filippenko (San Francisco: PASP Conf Ser, v.31)

Rieke, G.H. 1978, ApJ, 226, 550

Rieke, G.H., \& Lebofsky, M.J. 1979, ARA\&A, 17, 477

Rieke, G.H., \& Low, F.J. 1972, ApJ, 176, L95

Rieke, G.H., \& Low, F.J. 1975, ApJ, 200, L67

Rowan-Robinson, M., et al. 1991, Nature, 351, 719

Sanders, D.B., \& Mirabel, I.F. 1996, ARA\&A, 34, 749

Sanders, D.B., et al. 1989, ApJ, 347, 29

Sanders, D.B., Scoville, N.Z., \& Soifer, B.T. 1988c, ApJ, 335, L1

Sanders, D.B., et al. 1988b, ApJ, 328, L35

Sanders, D.B., et al. 1987, ApJ, 312, L5

Sanders, D.B., et al. 1988a, ApJ, 325, 74

Schmidt, M., \& Green, R.F. 1983, ApJ, 269, 352

Schmitt, H.R., Kinney, A.L., Calzetti, D., \& Storchi- Bergmann, T. 1997, AJ, 114,592

Scoville, N.Z., et al. 1989, ApJ, 345, L25

Smail, I., Ivison, R.J., \& Blain, A.W. 1997, ApJ, 490, L5

Stein, W.A., Gillett, F.C., Merrill, K.M. 1974, ApJ, 187, 213

Stockton, A., \& Ridgway, S.E. 1991, AJ, 102, 488

Surace, J.A., et al. 1998, ApJ, 492, 116

Taniguchi, Y., et al. 1997, A\&A, 328, L9

Telesco, C.M., \& Harper, D.A. 1980, ApJ, 235, 392

Ultraluminous Galaxies: Monsters or Babies 1998, Ap\&SS, in press

Vader, J.P., \& Simon, M. 1987, Nature, 327, 304

Veilleux, S., \& Osterbrock, D.E. 1987, ApJS, 63, 295

Veilleux, S., Kim, D.-C., \& Sanders, D.B. 1999, ApJ, in press 


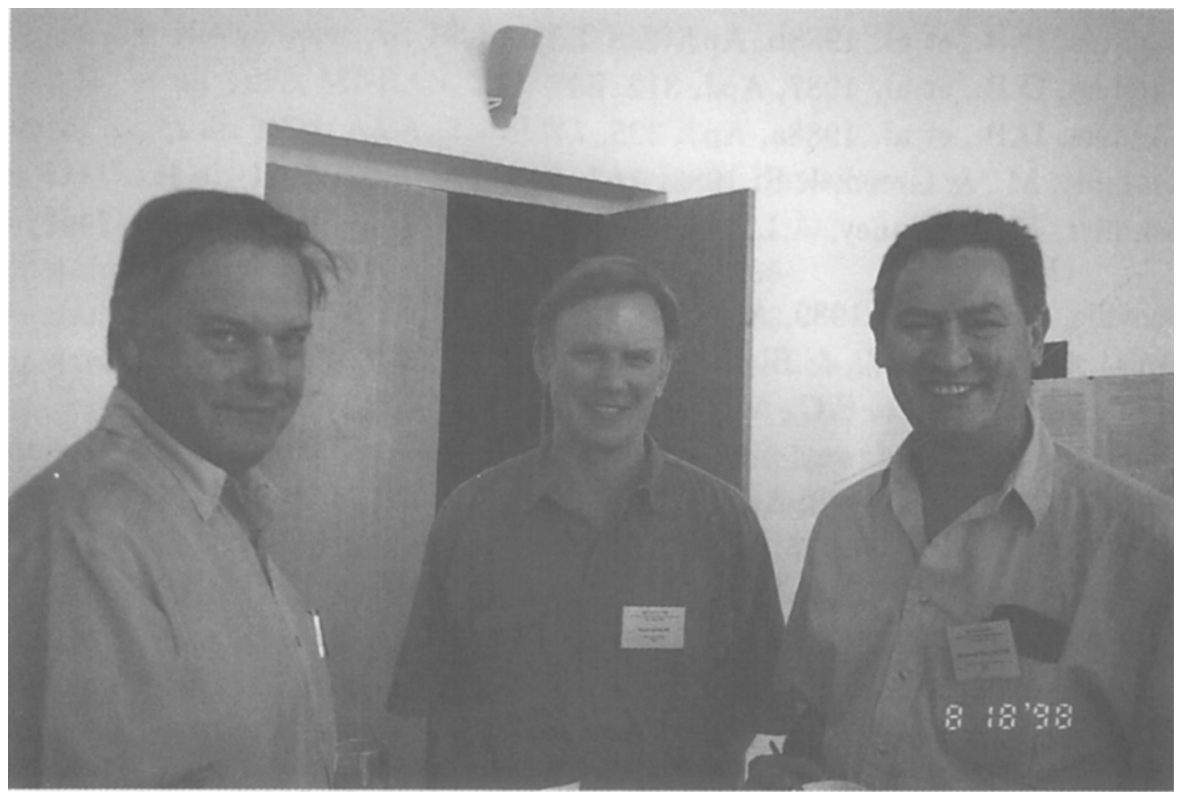

Gerard Leliévre, Dave Sanders and Duccio Macchetto 